\title{
Electronic and magnetic properties of a quasi-one-dimensional spin chain system, $\mathrm{Sr}_{3} \mathrm{NiRhO}_{6}$
}

\author{
S. K. Pandey and Kalobaran Mait* \\ Department of Condensed Matter Physics and Materials Science, \\ Tata Institute of Fundamental Research, Homi Bhabha Road, Colaba, Mumbai - 400 005, INDIA
}

(Dated: November 25, 2018)

\begin{abstract}
We investigate the electronic structure of $\mathrm{Sr}_{3} \mathrm{NiRhO}_{6}$, a quasi-one-dimensional spin chain system using $a b$ initio band structure calculations. Spin polarized calculations within GGA reveal that $\mathrm{Ni}$ and Rh have finite moments and they are antiferromagnetically coupled along the chain axis in the ground state. While these results obtained within the local spin density approximations provide remarkable representation of the magnetic phase, the experimentally observed insulating behavior could not be captured within this method. GGA $+U$ calculations show that opening up of an insulating gap requires on-site Coulomb interaction among the $\mathrm{Rh} 4 d$ electrons, $U_{d d}^{R h} \approx 2.5 \mathrm{eV}$ and the correlation among Ni $3 d$ electrons, $U_{d d}^{N i} \approx 4.5 \mathrm{eV}$ suggesting this system to be a Mott insulator. Electron correlation among $d$ electrons leads to significant enhancement of the $\mathrm{O} 2 p$ character in the energy bands in the vicinity of the Fermi level and the $d$ bands appear at lower energies. Energy gap in the up spin density of states appears to be significantly small $(\sim 0.12 \mathrm{eV})$ while it is $>$ $2 \mathrm{eV}$ in the down spin density of states suggesting possibility of spin polarized conduction in the semiconducting phase.

PACS numbers: 71.27.+a, 71.20.-b, 75.20.Hr
\end{abstract}

\section{INTRODUCTION}

Recently, quasi-one-dimensional compounds, $A E_{3} M M^{\prime} \mathrm{O}_{6} \quad(A E=$ alkaline earth metals such as $\mathrm{Ca}$, Sr etc.; $M$ and $M^{\prime}$ are transition metals) possessing rhombohedral $\mathrm{K}_{4} \mathrm{CdCl}_{6}$ structure (space group $R \overline{3} C$ ) have attracted a great deal of attention due to their fascinating magnetic properties, $, \frac{1,2}{2}, 3,4,5,6,7,8,9,10,11,12,13,14,15,16$ In this structure (see Fig. 1), $M \mathrm{O}_{6}$ forms in trigonal prismatic geometry and $M^{\prime} \mathrm{O}_{6}$ forms in octahedral geometry. These two units appear alternatively along the chain direction, $c$-axis and are connected via face sharing as shown in the figure. It is evident that the interchain interaction is significantly weak making these compounds a quasi-one-dimensional system. It is observed that antiferromagnetic, ferromagnetic or ferrimagnetic long-range ordered phases can be achieved by tuning the intrachain magnetic coupling in these one dimension chains $\frac{5,6,7}{}$ In addition to such interesting properties of the quasi-one-dimensional chains, the whole system can be viewed as made off antiferromagnetic or ferromagnetic chains arranged in a triangular lattice. Numerous studies based on tailoring the composition of these geometrically frustrated systems reveal plethora of novel phases such as spin liquid phase, partially disordered antiferromagnetic phase etc. $, 2,3,4,5,6$

In this paper, we report our results on the electronic structure of $\mathrm{Sr}_{3} \mathrm{NiRhO}_{6}$ using full potential linearized augmented plane wave method. This compound exhibits ferrimagnetic intrachain ordering below $45 \mathrm{~K}$. Further lowering in temperature leads to partially disordered antiferromagnetic phase below $10 \mathrm{~K} \cdot \underline{\underline{6}}$ Interestingly, analogous compound $\mathrm{Sr}_{3} \mathrm{NiPtO}_{6}$ exhibits spin-liquid behavior. While it is difficult to capture disorder effect and/or spin liquid phase using such $a b$ initio band structure calcula- tions, $\mathrm{Sr}_{3} \mathrm{NiRhO}_{6}$ is a good starting point to understand the intrachain coupling and associated ground state properties.

Our results clearly establish that the ground state of this compound consists of magnetic $\mathrm{Ni}$ and $\mathrm{Rh}$ ions which are antiferromagnetically coupled along the $c$-axis. To capture the insulating transport consistent with experimental observations, one needs to consider the on-site Coulomb interaction among $\mathrm{Ni} 3 d$ and $\mathrm{Rh} 4 d$ electrons in addition to the intrachain antiferromagnetic (IAFM) coupling, which suggests that this compound is a Mott insulator. On-site Coulomb interaction among Rh $4 d$ electrons is found to be lower (about 60\%) than that found for Ni $3 d$ electrons as expected due to larger radial extension of the Rh $4 d$ orbitals.

\section{COMPUTATIONAL DETAILS}

The spin polarized GGA (generalized gradient approximation) and GGA $+U$ ( $U=$ on-site Coulomb repulsion strength) electronic structure calculations were carried out using state-of-the art full potential linearized augmented plane wave (FPLAPW) method $\stackrel{17}{1}$ The lattice parameters and atomic positions used in the calculations are taken from literature $\underline{\underline{8}}$ The Muffin-Tin sphere radii were chosen to be $2.33,2.19,2.01$, and 1.78 a.u. for $\mathrm{Sr}$, $\mathrm{Ni}, \mathrm{Rh}$, and $\mathrm{O}$ atoms, respectively. For the exchange correlation functional, we have adopted recently developed

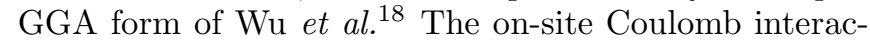
tions were considered within $\mathrm{LSDA}+U(\mathrm{LSDA}=$ local spin density approximation) formulation of Anisimov et $a l .19$ The calculations were performed by varying on-site Coulomb interaction among $\mathrm{Ni} 3 d$ electrons, $U_{d d}^{N i}$ and $\mathrm{Rh}$ $4 d$ electrons, $U_{d d}^{R h}$. The convergence was achieved by con- 


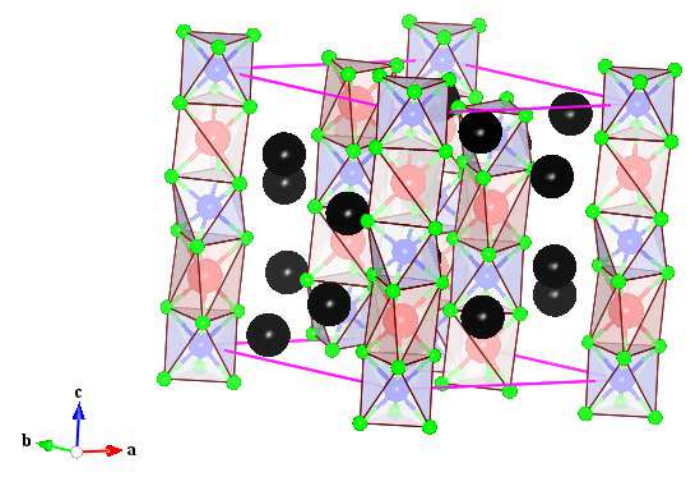

FIG. 1: (Color online) The unit cell of $\mathrm{Sr}_{3} \mathrm{NiRhO}_{6}$. $\mathrm{Sr}$ is represented by largest symbols (black). Gradually decreasing sized symbols represent $\mathrm{Ni}$ (pink), Rh (blue) and O (green), respectively.

sidering $512 k$ points within the first Brillouin zone and the error bar for the energy convergence was set to be smaller than $10^{-4} \mathrm{Ryd} /$ cell.

\section{RESULTS AND DISCUSSIONS}

The crystal structure of $\mathrm{Sr}_{3} \mathrm{NiRhO}_{6}$ is hexagonal as shown in Fig. 1, where the $\mathrm{Sr}, \mathrm{Ni}, \mathrm{Rh}$, and $\mathrm{O}$ ions are denoted by spheres of decreasing size. It is evident from the figure that the $\mathrm{Ni}$ and $\mathrm{Rh}$ ions are surrounded by six oxygen ions forming trigonal prism and octahedron, respectively. The trigonal prisms and octahedra are connected by face sharing and form the chains along the $c$-axis. If the axis system is defined such that $z$-axis is along $c$-axis and $x$ - and $y$-axis are in the $a b$-plane, both $\mathrm{Ni} 3 d$ and $\mathrm{Rh} 4 d$ orbitals defined in this axis system will not be degenerate due to the corresponding crystal field effect. There will be three bands consisting of $d_{0}, d_{ \pm 1}$ and $d_{ \pm 2}$ orbitals. The energy separation and the location of the bands in the energy axis will depend on the type of crystal field is applicable as discussed later in the text.

In Fig. 2, we show the partial density of states (PDOS)

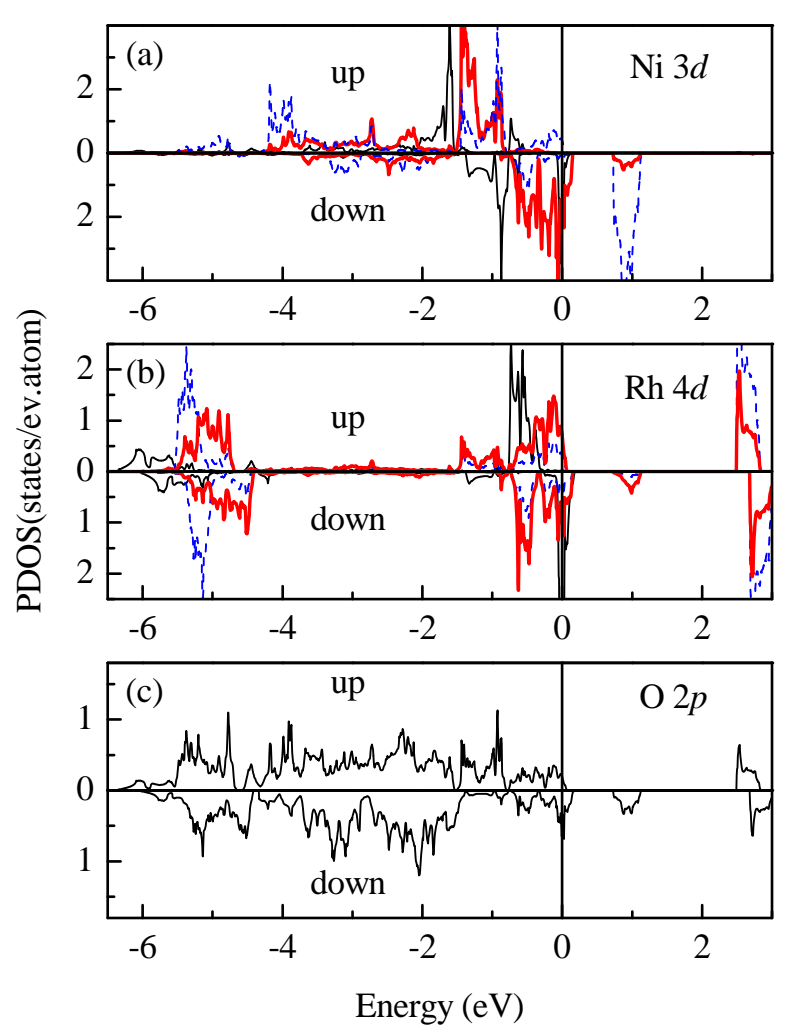

FIG. 2: Color online) The partial density of states (PDOS) corresponding to $d_{0}$ (thin solid lines), $d_{ \pm 1}$ (dashed lines) and $d_{ \pm 2}$ (thick solid lines) orbitals for (a) Ni and (b) Rh obtained from spin-polarized GGA calculation when $\mathrm{Ni}$ and $\mathrm{Rh}$ are ferromagnetically coupled. (c) O $2 p$ PDOS.

corresponding to Ni $3 d$, Rh $4 d$ and O $2 p$ electronic states obtained from GGA calculations, where $\mathrm{Ni}$ and $\mathrm{Rh}$ are ferromagnetically coupled. The band splitting due to the crystal field as discussed above is visible clearly in the figure. In Fig. 2(a), the energy range above $-2 \mathrm{eV}$ is essentially contributed by Ni $3 d$ electronic states. The lower energy region $(-4$ to $-2 \mathrm{eV})$ contains very low intensity of $\mathrm{Ni} 3 d \mathrm{PDOS}$ and has dominant $\mathrm{O} 2 p$ contributions. The energy distributions of the density of states (DOS) corresponding to both Ni $3 d$ and O $2 p$ states appear similar. These results suggest that the DOS in -4 to $-2 \mathrm{eV}$ range can be attributed to bonding states arising from $\mathrm{Ni} 3 d$ and $\mathrm{O} 2 p$ hybridizations and the antibonding states with dominant Ni $3 d$ character appear above $-2 \mathrm{eV}$.

The DOS in the energy ranges -6.3 to $-4.4 \mathrm{eV}$ and -1.5 to $0 \mathrm{eV}$ correspond to $\mathrm{Rh} 4 d$-O $2 p$ bonding and antibonding bands, respectively. The intensity of $\mathrm{Rh} 4 d$ PDOS is almost similar in these two regions, with negligible con- 
tribution in the energy region of -4.4 to $-1.5 \mathrm{eV}$ (see Fig. 2(b)). Two distinct features are observed in Fig. 2. (i) The energy separation between bonding and antibonding bands arising due to $\mathrm{Ni} 3 d-\mathrm{O} 2 p$ hybridizations is significantly smaller than the energy separation between the bonding and antibonding Rh $4 d-\mathrm{O} 2 p$ bands. (ii) The bonding and antibonding $\mathrm{Ni} 3 d-\mathrm{O} 2 p$ bands have dominant $\mathrm{O} 2 p$ and $\mathrm{Ni} 3 d$ character, respectively. However, the mixing of Rh $4 d$ and $\mathrm{O} 2 p$ characters in the $\mathrm{Rh} 4 d-\mathrm{O}$ $2 p$ hybridization is much stronger. These observations clearly demonstrate the stronger $\mathrm{O} 2 p$ - $\mathrm{Rh} 4 d$ hybridization presumably due to larger radial extension of the $4 d$ orbitals compared to the $3 d$ orbitals. Such large overlap integral, $t$ leads to larger separation of the bonding and antibonding bands. However, the bandwidth of the individual bands does not appear to increase.

In addition, it is evident from Fig. 2(a) that up-spin channel of $\mathrm{Ni} 3 d$ states is almost fully occupied, whereas down-spin channel is partially occupied. Major contribution in the unoccupied level comes from $d_{ \pm 1}$ orbitals. In the case of Rh $4 d$ states, DOS corresponding to both the spin states are partially occupied and thus, contribute to the unoccupied DOS.

The GGA calculations for ferromagnetic (FM) intrachain coupling converged to the metallic ground state in sharp contrast to the experimentally observed insulating behavior in this compound. Moreover, the total magnetic moment per formula unit (fu) obtained from this calculation is about $2.85 \mu_{B}$, which is much larger than the experimentally estimated value of $\sim 1 \mu_{B}, \underline{6}$ The magnetic moment centered at $\mathrm{Ni}$ and $\mathrm{Rh}$ sites are $1.55 \mu_{B}$ and $0.43 \mu_{B}$, respectively. Magnetic moment centered at the oxygen sites is found to be $0.13 \mu_{B}$, which is large and induced by the $\mathrm{Ni} 3 d$ and $\mathrm{Rh} 4 d$ moments.

It is evident from the magnetic moments that if $\mathrm{Ni}$ and Rh are antiferromagnetically coupled, the total magnetic moment would match with the experimental results. In order to investigate such possibility, we have calculated the ground state energies and wavefunctions corresponding to antiferromagnetic coupling between $\mathrm{Ni} 3 d$ and $\mathrm{Rh}$ $4 d$ moments. Interestingly, the calculated ground state energy is found to be $27 \mathrm{meV} / \mathrm{fu}$ lower than that of FM state. The calculated total magnetic moment comes out to be $1.04 \mu_{B} / \mathrm{fu}$. Both these findings provide remarkable representation of the experimental magnetization data 6 The calculated magnetic moments for $\mathrm{Ni}$ and Rh are 1.31 $\mu_{B}$ and $-0.36 \mu_{B}$, respectively. The moment centered at oxygen site is almost negligible $\left(0.02 \mu_{B}\right)$.

The calculated Ni $3 d$, Rh $4 d$ and O $2 p$ PDOS are shown in Fig. 3. It is evident from the figure that intrachain antiferromagnetic interaction leads to significant reduction in bandwidth of all the energy bands and redistribution in spectral weight. For example, Ni $3 d$ up spin band is almost completely filled and the Fermi level, $\epsilon_{F}$ is pinned at the top of the down spin band having dominant $d_{ \pm 2}$ contributions and a width of about $0.5 \mathrm{eV}$ while $\mathrm{Ni} 3 d$ down spin band in Fig. 2 has a width of about $1 \mathrm{eV}$ and is partially filled. The occupancy of $\mathrm{Rh} 4 d$ up spin and

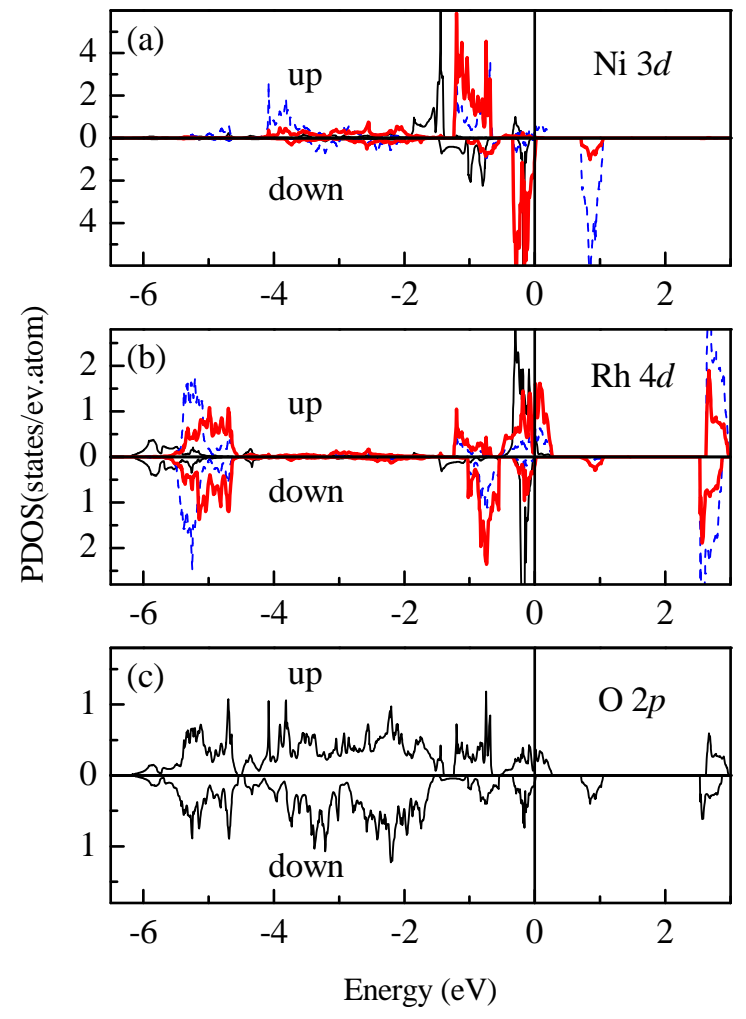

FIG. 3: (Color online) The partial density of states (PDOS) corresponding to $d_{0}$ (thin solid lines), $d_{ \pm 1}$ (dashed lines) and $d_{ \pm 2}$ (thick solid lines) orbitals for (a) Ni and (b) Rh obtained from spin-polarized GGA calculation when intrachain $\mathrm{Ni}$ and $\mathrm{Rh}$ are antiferromagnetically coupled. (c) O $2 p$ PDOS.

down spin PDOS exhibit spectral distribution opposite to that observed in Fig. 2 as expected due to antiferromagnetic coupling. In this case also the bands are narrower than those observed in Fig. 2. The $\mathrm{O} 2 p$ contribution at the Fermi level is dominant for up spin while in the ferromagnetic case it was down spin.

All the above results clearly establish that the LSDA approach is adequate to capture the magnetic phase in this system. Although, there is significant spectral weight redistributions observed for different magnetic configurations, both these calculations converge to metallic ground state in contrast to the experimental studies.

It is well known that such $a b$ initio calculations often underestimate the on-site Coulomb correlation energy, which plays significant role in determining the electronic properties of $d$ and $f$ electron systems ${ }^{20}$ The consideration of on-site Coulomb interaction among the $d$ electrons $\left(U_{d d}\right)$ under GGA $+U$ formulation is expected to improve 
the situation. Thus, we have carried out GGA $+U$ calculations for different values of $U_{d d}$ corresponding to $\mathrm{Ni}$ $3 d$ electrons, which is defined as $U_{d d}^{N i}$ and $\mathrm{Rh} 4 d$ electrons, which is defined as $U_{d d}^{R h}$. The calculations were carried out considering the intrachain antiferromagnetic coupling.

The calculated band gaps in the vicinity of $\epsilon_{F}$ for up spin and down spin density of states are shown in Fig. 4. It is evident from the figure that consideration of the electron correlation for both $\mathrm{Ni} 3 d$ and Rh $4 d$ electrons helps to achieve the insulating phase. We have shown two types of results in the figure, (i) the dependence of the band gap as a function of $U_{d d}^{N i}$ for a fixed value of $U_{d d}^{R h}=3 \mathrm{eV}$ and (ii) band gap as a function of $U_{d d}^{R h}$ for a fixed value of $U_{d d}^{N i}=5.5 \mathrm{eV}$. In the up spin channel (see Fig. 4(a)), it is evident that an increase in $U_{d d}^{N i}$ does not lead to insulating phase till $U_{d d}^{N i}=4 \mathrm{eV}$. An increase in $U_{d d}^{N i}$ above $4 \mathrm{eV}$ provides an energy gap of about 0.12 $\mathrm{eV}$. Interestingly, the band gap remains unchanged for further increase in $U_{d d}^{N i}$. On the other hand, the calculations as a function of $U_{d d}^{R h}$ for $U_{d d}^{N i}=5.5 \mathrm{eV}$, indicate gradual increase in band gap. Thus, the energy gap is essentially determined by $U_{d d}^{R h}$ when $U_{d d}^{N i}$ is kept large. The Fig. 4(a) suggests that the creation of insulating ground state requires $U_{d d}^{R h}$ to be at least $2.6 \mathrm{eV}$. Since the Coulomb repulsion strength depends inversely on the separation of the two electrons, the strength of on-site Coulomb interaction is sensitive to the radial extension of the wave functions. The present result of $U_{d d}^{N i}$ larger than $U_{d d}^{R h}$ is consistent with this behavior.

Calculations for the down spin channel exhibit gradual increase in band gap with the increase in $U_{d d}$ for both $\mathrm{Ni}$ $3 d$ and $\mathrm{Rh} 4 d$ electrons. The magnitude of the gap in this case is always much higher $(>2 \mathrm{eV})$ than that $(<1$ $\mathrm{eV}$ ) observed for up spin density of states. It is thus clear that various electronic properties of this compound will essentially be controlled by electronic density of states in the up spin channel.

In order to investigate the character of the electronic states in the valence band, we show the calculated PDOS for $U_{d d}^{N i}=5 \mathrm{eV}$ and $U_{d d}^{R h}=3 \mathrm{eV}$ in Fig. 5. All the bands are significantly modified due to the spectral weight transfer from $\epsilon_{F}$ to the energy region away from it. Ni $3 d_{ \pm 1}$ contributions exhibit significant band narrowing. The up spin bands appear around $-5.8 \mathrm{eV}$ and the down spin ones around $2.5 \mathrm{eV}$. The contributions from other $\mathrm{Ni}$ $3 d$ orbitals appear between $-2 \mathrm{eV}$ to $-5 \mathrm{eV}$. Interestingly, the consideration of $U_{d d}^{N i}$ leads to a significant increase of $\mathrm{Ni} 3 d$ character at higher energies. Thus the O $2 p$ contributions relative to $\mathrm{Ni} 3 d$ become significantly high in the vicinity of the Fermi level.

Consideration of the on-site Coulomb interaction among Rh $4 d$ electrons reveals large effect on the density of states distribution. In the GGA calculation $d_{0}$ was almost fully occupied and there was a significant contribution of $d_{ \pm 1}$ and $d_{ \pm 2}$ orbitals within $0.28 \mathrm{eV}$ of $\epsilon_{F}$ in the unoccupied up spin channel as evident from Fig. $3(\mathrm{~b})$. This trend is reversed in $\mathrm{GGA}+U$ calculations,
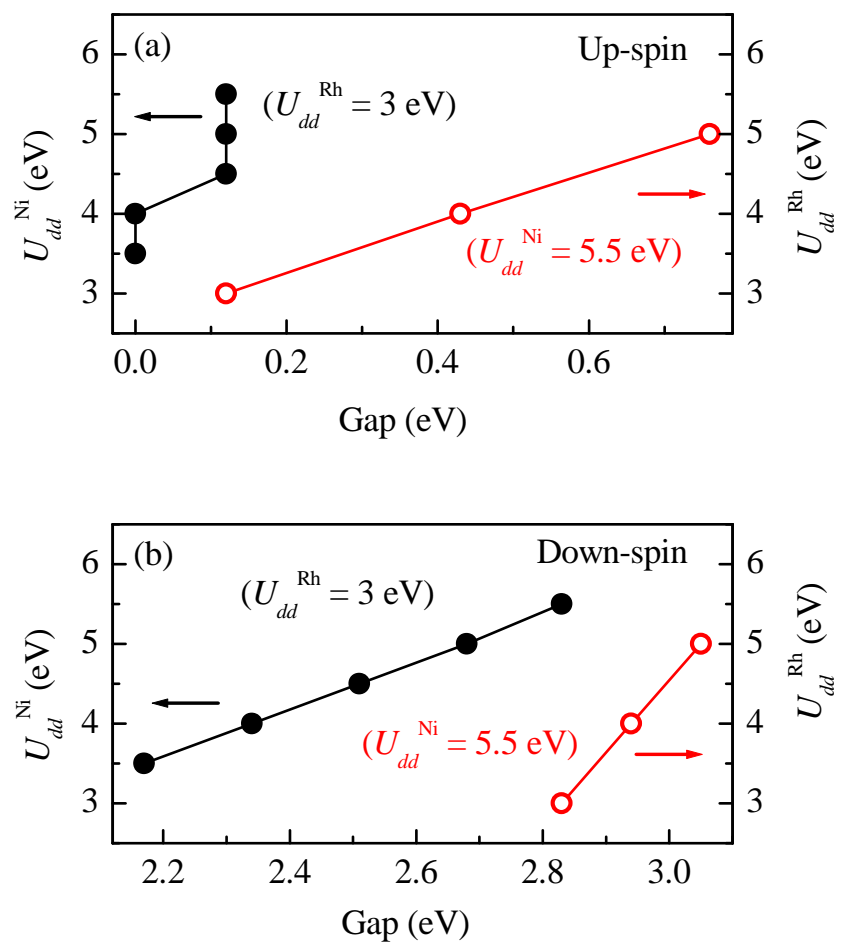

FIG. 4: (Color online) On-site Coulomb interaction among $\mathrm{Ni} 3 d\left(U_{d d}^{N i}\right)$ and $\mathrm{Rh} 4 d\left(U_{d d}^{R h}\right)$ electrons vs. insulating gap for (a) up-spin and (b) down-spin channels. Two cases are considered: (i) $U_{d d}^{R h}$ is fixed at $3 \mathrm{eV}$ and $U_{d d}^{N i}$ varied from 3.5 to $5.5 \mathrm{eV}$ (solid circles) and (ii) $U_{d d}^{N i}$ is fixed at $5.5 \mathrm{eV}$ and $U_{d d}^{R h}$ varied from 3 to $5 \mathrm{eV}$ (open circles).

where region within $0.75 \mathrm{eV}$ above the $\epsilon_{F}$ is contributed by the electronic states having $d_{0}$ character. The electronic states below $\epsilon_{F}$ are contributed by $d_{ \pm 1}$ and $d_{ \pm 2}$ orbitals.

Despite the fact that the Ni $3 d$ PDOS appear far away from $\epsilon_{F}$, it has finite contribution in the vicinity of $\epsilon_{F}$. This is evident in the rescaled Ni $3 d \operatorname{PDOS}(\times 15+1)$ shown in Fig. 5(a) by dot-dashed line. Interestingly, the energy distribution of these density of states closely follows the distribution observed in Rh $4 d$ and O $2 p$ PDOS. This clearly manifests the effect of hybridization between $\mathrm{Ni} 3 d$ and $\mathrm{Rh} 4 d$ states via $\mathrm{O} 2 p$ states. Most interestingly, the gap in the up spin channel is much smaller than that found in down spin channel. Thus, this system provides an unique example of a semiconductor, where the electronic conduction is spin polarized. While halfmetallic materials have long been investigated due to spin polarized conduction of charge carriers in this system. 


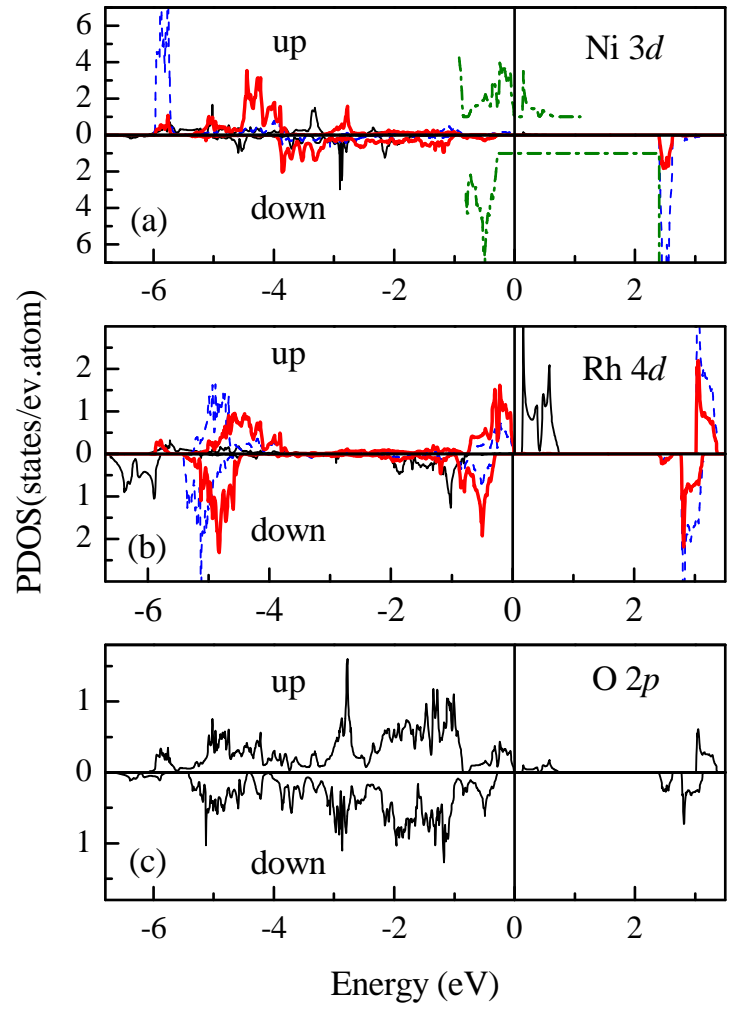

FIG. 5: (Color online) The partial density of states (PDOS) corresponding to $d_{0}$ (thin solid lines), $d_{ \pm 1}$ (dashed lines) and $d_{ \pm 2}$ (thick solid lines) orbitals for (a) Ni and (b) Rh obtained from spin-polarized GGA $+U$ calculation $\left(U_{d d}^{N i}=5 \mathrm{eV}\right.$ and $U_{d d}^{R h}=3 \mathrm{eV}$ ) when intrachain $\mathrm{Ni}$ and $\mathrm{Rh}$ are antiferromagnetically coupled. (c) O $2 p$ PDOS.

This compound manifests properties that can be more useful in the semiconductor industry, where spin based technology is envisaged.

Now, we discuss the manifestation of intrachain antiferromagnetic coupling and Coulomb correlation in the band structure along different high symmetry directions of the Brillouin zone. The dispersion curves corresponding to different bands for FM, IAFM and IAFM considering $U_{d d}^{N i}=5$ and $U_{d d}^{R h}=3 \mathrm{eV}$ for both the spin channels are plotted in Fig. 6. The zero in the energy scale indicates $\epsilon_{F}$. In the ferromagnetic solution, the up spin bands along $\Gamma M$ and $\Gamma A$ directions do not cross the Fermi level, while the down spin bands cross $\epsilon_{F}$ in all the directions indicating half metallicity along these directions. The antiferromagnetic coupling among $\mathrm{Ni}$ and $\mathrm{Rh}$ in the chains leads to narrowing of the bands and the up spin bands cross the Fermi level in all the directions. On the other hand the down spin bands in the vicinity of $\epsilon_{F}$
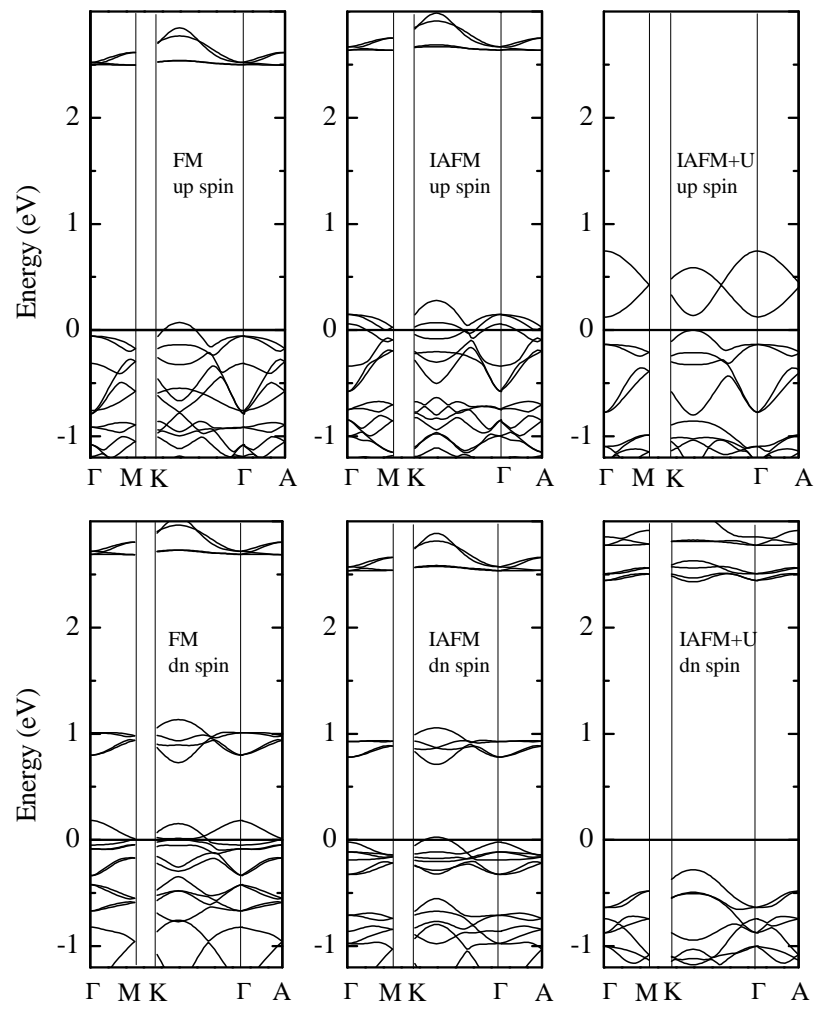

FIG. 6: Dispersion curves along different symmetry directions of the first Brillouin zone for both the spin channels. Fermi level is denoted by zero in the energy scale.

essentially appear below $\epsilon_{F}$. Again, the band crossing of the down spin band is observed only along $K \Gamma$ direction suggesting half metallicity in other directions.

Inclusion of the electron correlation among the $d$ electrons leads to significant change in the band structure. While, there is a hard gap in both the spin channels consistent with the transport behavior of this compound, the gap exhibit significant $k$-dependence. The gap at the high symmetry point, $\Gamma$ is lower than that observed at $M$, $K$ or $A$ points. Interestingly, the dispersion of the bands close to $\epsilon_{F}$ appears to be larger than that observed in the uncorrelated case. This indicates that although electron correlation essentially tends to localize the electrons, the electronic states in the vicinity of the Fermi level are highly mobile.

The occupancies of different $d$ orbitals corresponding to $\mathrm{Ni}$ and Rh obtained from $\mathrm{GGA}+U\left(U_{d d}^{N i}=5\right.$ and $U_{d d}^{R h}$ $=3 \mathrm{eV}$ ) calculation are shown in Table 1 . In up spin channel, all the Ni $3 d$ orbitals are almost completely filled and in down spin channel, only $d_{0}$ orbital is completely 
filled. The small deviations $(\sim 0.05)$ from the completely filled values can be attributed to the hybridization of $\mathrm{Ni}$ $3 d$ orbitals with the neighboring $\mathrm{O} 2 p$ orbitals. Total number of electron in the Ni $3 d$ is found to be 7.91, which suggests that the valency of $\mathrm{Ni}$ is close to $2+$.

Table 1: The electron occupancies of $d_{0}, d_{ \pm 1}$, and $d_{ \pm 2}$ orbitals for $\mathrm{Ni}$ and $\mathrm{Rh}$ obtained from spin polarized $\mathrm{GGA}+U$ calculation.

\begin{tabular}{|c|c|c|c|c|c|c|}
\hline \hline & $d_{-2}$ & $d_{-1}$ & $d_{0}$ & $d_{1}$ & $d_{2}$ & Total \\
\hline Ni-up & 0.95 & 0.98 & 0.94 & 0.98 & 0.95 & 4.8 \\
\hline Ni-down & 0.8 & 0.28 & 0.95 & 0.28 & 0.8 & 3.11 \\
\hline Rh-up & 0.65 & 0.5 & 0.21 & 0.5 & 0.65 & 2.51 \\
\hline Rh-down & 0.66 & 0.52 & 0.82 & 0.52 & 0.66 & 3.18 \\
\hline \hline
\end{tabular}

In the case of $\mathrm{Rh} 4 d$ orbitals, both the spin channels are partially occupied. Our calculations exhibit 5.69 electrons in the $\mathrm{Rh} 4 d$ orbitals. If we consider $\mathrm{O}$ in 2 - and $\mathrm{Sr}$ in $2+$ states, then charge neutrality condition demands $\mathrm{Rh}$ to be in $4+$ state, which corresponds to 5 electrons in the $4 d$ orbitals. The extra 0.69 electrons can be attributed to strong hybridization of $\mathrm{Rh} 4 d$ orbitals with the neighboring $\mathrm{O} 2 p$ orbitals.

The calculated magnetic moments for $\mathrm{Ni}$ and $\mathrm{Rh}$ ions are $1.69 \mu_{B}$ and $-0.68 \mu_{B}$, respectively, with almost negligible contribution from oxygen $\left(0.01 \mu_{B}\right)$. The total magnetic moments per formula unit, including the contribution of $-0.07 \mu_{B}$ from the interstitial region, is found to be about $1 \mu_{B}$ consistent with the experimental results $\frac{6}{6}$

In order to understand the possible cause for IAFM interaction between $\mathrm{Ni}$ and $\mathrm{Rh}$ spins, we show the schematic crystal field split energy level diagram for both $\mathrm{Ni}$ and $\mathrm{Rh}$ obtained from spin unpolarized GGA calculation by considering centers of gravity of $d_{0}, d_{ \pm 1}$, and $d_{ \pm 2}$ PDOS is shown in Fig. 7. It is evident from the figure that $d_{0}$ orbital has lowest energy for both $\mathrm{Ni}$ and $\mathrm{Rh}$. However, next energy levels consist of different orbitals. In $\mathrm{Ni}$, next level corresponds to $d_{ \pm 1}$ orbitals, whereas in $\mathrm{Rh}$ it is $d_{ \pm 2}$ orbitals. The separation of $\mathrm{Rh} d_{ \pm 2}$ and $d_{ \pm 1}$ is found to be $\sim 0.7 \mathrm{eV}$, which is about double of the Hund's coupling energy $\left(J_{H}\right)$ of Rh $4 d$ electrons estimated from spin polarized GGA calculation. On the other hand the energy separation of $\mathrm{Ni} d_{0}, d_{ \pm 1}$, and $d_{ \pm 2}$ orbitals are found to be $\sim 0.3 \mathrm{eV}$, which is about one fifth of the estimated value of $J_{H}$ for Ni $3 d$ electrons.

As discussed above, $\mathrm{Ni}$ is in $2+$ state leading to $S=$ 1 state. $\mathrm{Rh}^{4+}$ has 5 electrons in the $4 d$ orbitals. The schematic diagram clearly shows that $\mathrm{Rh}$ atoms will be in low-spin $(S=1 / 2)$ state with 2 electrons occupying $d_{0}$ orbital and the other 3 electrons will occupy $d_{ \pm 2}$ orbitals making them more than half-filled. It is well known that in such a case, the interacting electrons via oxygen $2 p$ orbitals (superexchange interaction) favor antiparallel coupling in the ground state $\stackrel{21}{1}^{2}$

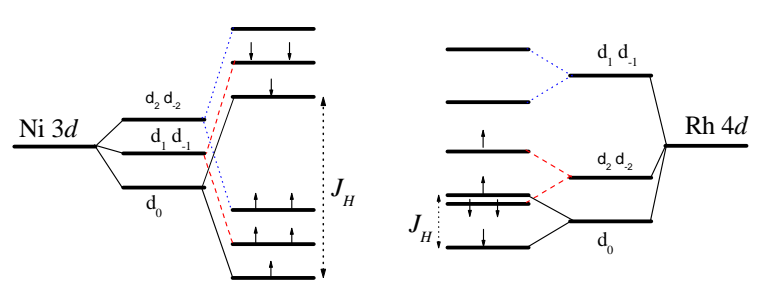

FIG. 7: (Color online) Schematic energy level diagram of $d_{0}$ ,$d_{ \pm 1}$, and $d_{ \pm 2}$ orbitals for $\mathrm{Ni}^{2+}$ and $\mathrm{Rh}^{4+}$ showing antiferromagnetic coupling as the possible ground state.

\section{CONCLUSIONS}

In conclusion, we studied the electronic and magnetic properties of $\mathrm{Sr}_{3} \mathrm{NiRhO}_{6}$ using ab initio spin polarized band structure calculations within GGA and GGA $+U$. The calculations for intrachain antiferromagnetic coupling exhibit the lowest energy. The magnetic moments of $\mathrm{Ni}$ and $\mathrm{Rh}$ ions were found to be $\sim 1.7 \mu_{B}$ and -0.7 $\mu_{B}$, respectively, with a total moment of about $1 \mu_{B}$. All these results corresponding to the magnetic phase provide a remarkable representation of the experimental results.

The insulating phase observed in transport measurements could not be captured within GGA suggesting importance of electron correlation. GGA $+U$ calculation reveals that the critical values of Coulomb correlation strength to open up the insulating gap are $\sim 4.5$ and $\sim 2.5 \mathrm{eV}$ for $\mathrm{Ni} 3 d$ and $\mathrm{Rh} 4 d$ electrons, respectively. The inclusion of electron correlation leads to significant change in character of the energy bands. The feature close to $\epsilon_{F}$ exhibits enhancement of $\mathrm{O} 2 p$ character, while the $d$ character enhances at higher energies. Interestingly, the band gap in up spin channel is found to be significantly small $(\sim 0.12 \mathrm{eV})$ compared to that observed $(>$ $2 \mathrm{eV}$ ) in the down spin channel indicating possibilities of spin polarized conduction in the semiconducting phase.

\section{ACKNOWLEDGEMENTS}

The authors acknowledge Prof. E.V. Sampathkumaran, TIFR, India for drawing our attention towards 
this compound and useful discussions.

* Electronic mail: kbmaiti@tifr.res.in

1 S. Niitaka, H. Kageyama, M. Kato, K. Yoshimura, and K. Kosuge, J. Solid State Chem. 146, 137 (1999).

2 E. V. Sampathkumaran and A. Niazi, Phys. Rev. B 65, 180401(R) (2002).

3 D. Flahaut, S. Hébert, A. Maignan, V. Hardy, C. Martin, M. Hervieu, M. Costes, B. Raquet, and J.M. Broto, Eur. Phys. J B 35, 317 (2003).

4 S. Agrestini, C. Mazzoli, A. Bombardi, and M.R. Lees, arXiv:0801.2495 1.

5 S. Niitaka, K. Yoshimura, K. Kosuge, M. Nishi, and K. Kakurai, Phys. Rev. Lett. 87, 177202 (2001).

6 N. Mohapatra, K. K. Iyer, S. Rayaprol, and E. V. Sampathkumaran, Phys. Rev. B 75, 214422 (2007).

7 H. Wu, Z. Hu, D. I. Khomskii, and L. H. Tjeng, Phys. Rev. B 75, 245118 (2007).

${ }^{8}$ K. E. Stitzer, W. H. Henley, J. B. Claridge, H. -C. zur Loye, and R. C. Layland, J. Solid State Chem. 164, 220 (2002).

${ }^{9}$ K. Sengupta, S. Rayaprol, K. K. Iyer, and E. V. Sampathkumaran, Phys. Rev. B 68, 012411 (2003).

10 M. -H. Whangbo, D. Dai, H. -J. Koo, and S. Jobic, Solid State Commun. 125413 (2003).

11 R. Vidya, P. Ravindran, H. Fjellvàg, and A. Kjekshus, Phys. Rev. Lett. 91, 186404 (2003).

12 E. V. Sampathkumaran, N. Fujiwara, S. Rayaprol, P. K.
Madhu, and Y. Uwatoko, Phys. Rev. B 70, 014437 (2004).

13 R. Frésard, C. Laschinger, T. Kopp, and V. Eyert, Phys. Rev. B 69, 140405(R) (2004).

${ }^{14} \mathrm{H}$. Wu, M. W. Haverkort, Z. Hu, D. I. Khomskii, and L. H. Tjeng, Phys. Rev. Lett. 95, 186401 (2005).

15 K. Takubo, T. Mizokawa, S. Hirata, J.-Y. Son, A. Fujimori, D. Topwal, D. D. Sarma, S. Rayaprol, and E.-V. Sampathkumaran, Phys. Rev. B 71, 073406 (2005).

16 J. Sugiyama, H. Nozaki, Y. Ikedo, P. L. Russo, K. Mukai, D. Andreica, A. Amato, T. Takami, and H. Ikuta, Phys. Rev. B 77, 092409 (2008).

17 P. Blaha, K. Schwarz, G.K.H. Madsen, D. Kvasnicka, and J. Luitz, WIEN2k, An Augmented Plane Wave + Local Orbitals Program for Calculating Crystal Properties (Karlheinz Schwarz, Techn. Universität Wien, Austria), 2001. ISBN 3-9501031-1-2.

18 Z. Wu and R. E. Cohen, Phys. Rev. B 73, 235116 (2006).

19 V. I. Anisimov, I. V. Solovyev, M. A. Korotin, M. T. Czyżyk and G. A. Sawatzky, Phys. Rev. B 48, 16929 (1993).

20 V. I. Anisimov, F. Aryasetiawan, and A. I. Lichtenstein, J. Phys.: Condens. Matter 9, 767 (1997).

21 J. B. Goodenough, in Magnetism and the Chemical Bond (Interscience Publishers, New York-London, 1963). 